\title{
Intestinal microbiota transplant - current state of knowledge
}

\author{
Jarosław Jerzy Leszczyszyn ${ }^{1,2}$, Marek Radomski ${ }^{3,4}$, Anna Maria Leszczyszyn ${ }^{5}$ \\ ${ }^{1}$ National Institute of Geriatrics, Rheumatology and Rehabilitation, Warsaw, Poland \\ ${ }^{2}$ Biotransplant Research, Wroclaw, Poland \\ ${ }^{3}$ National Institute of Geriatrics, Rheumatology and Rehabilitation, Warsaw, Poland \\ ${ }^{4}$ School of Pharmacy and Pharmaceutical Sciences, University of Dublin, Trinity College, Dublin, Ireland \\ ${ }^{5}$ Department of Oral Surgery, Medical University in Wroclaw, Wroclaw, Poland
}

\begin{abstract}
Faecal microbiota transplantation (FMT) has induced a lot scientific interest and hopes for the last couple of years. FMT has been approved as a treatment of recurrent Clostridium difficile colitis. Highly sophisticated molecular DNA identification methods have been used to assess the healthy human microbiome as well as its disturbances in several diseases. The metabolic and immunologic functions of the microbiome have become more clear and understandable. A lot of pathological changes, such as production of short-chain fatty acids or components of the inflammatory cascade, caused by changes in microbiome diversity, variability and richness have been observed among patients suffering from inflammatory bowel diseases, irritable bowel syndrome, type 2 diabetes or rheumatoid arthritis. The published clinical results are encouraging, but still there is huge demand for FMT controlled clinical trials.
\end{abstract}

Key words: faecal microbiota transplantation, microbiome, inflammatory bowel disease.

In recent years, due to the numerous publications on the research of the human intestinal microbiome, the possibility of permanent modification of the microbiome through faecal microbiota transplantation (FMT) from healthy to ill individuals has become a subject of increased attention. It has been mainly the result of numerous publications confirming good results of applying FMT in the treatment of Clostridium difficile infections [1]. In 2014 use of FMT in Clostridium difficile infections coexisting with inflammatory bowel disease was included in the treatment standards of the European Crohn's and Colitis Organization [2].

The natural human intestinal microbiome constitutes a diverse biological environment, being shaped and stabilized from the moment of birth. The microbiological environment of the intestines is very complex and develops with age. Immediately after birth, this environment is very changeable and susceptible to contact with environmental stimuli and food. Approximately at the age of 3 years, this environment stabilizes, becoming similar to that of the adult human [3]. In elderly adults the microbiological intestinal environment once again, just like after birth, becomes susceptible to the influence of external factors and pathogens [4] - primarily due to its limited biodiversity. The reduction in diversity is considered a major cause of Clostridium difficile infections affecting elderly individuals. The microbiome diversity and variety are influenced by a number of factors, such as diet, domicile or the way of life and feeding habits associated with urban or rural lifestyle [5]. Yatsushenko et al. [3] demonstrated that the intestinal microbiome of US inhabitants is far less diverse than that of individuals living in other, less developed countries. Multiple factors can be considered as a cause of such a situation, including the growing number of caesarean sections in the American population, depriving newborns of natural contact with the bacterial environment of the vagina [6] and the common decision not to breastfeed, leading

Address for correspondence:

Jarosław Jerzy Leszczyszyn, National Institute of Geriatrics, Rheumatology and Rehabilitation, Spartańska 1, 02-637 Warsaw, Poland,

e-mail: j.leszczyszyn@melitamedical.pl

Submitted: 29.01.2016; Accepted: 26.02.2016 
to the replacement of mother's milk with sterile food based on cow's milk [7]. The Western diet, rich in highly processed, ready-made, sterile dishes with preservatives added [8], use of water treated with oxygen- and chlorine-derived disinfectants and the common antibiotic use without absolute indications for such therapy [9] all play a significant role as well.

The intestinal microbiome consists of numerous bacteria, viruses and fungi living in the intestinal contents (faecal mass), as well as in the mucus covering the intestinal mucosa. It has been considered that these two habitats constitute two separate microbiological commensal ecosystems, having different functions in the interference between them and the organism of the host. Bacteria associated with the intestinal contents play mostly a metabolic role, utilizing large quantities of substrates contained in the intestinal contents. The bacteria of the mucous layer mainly take part in the regulation of the immunological processes in the mucosa and in the metabolic and immunological communication with the host $[10,11]$. Bacterial obligate anaerobes dominate the biological environment of the intestines. It is presumed that they are responsible to a large extent for restraining the colonization of the intestine by other bacteria [12]. Currently, over 1000 genera of intestinal bacteria have been identified [13].

It should be noted that the exploration of this ecosystem, which coexists with the human organism, became possible only after the introduction of molecular DNA research techniques. The most common method of DNA testing used in human microbiome research is $16 \mathrm{~S}$ ribosomal RNA (rRNA) sequencing [14]. This unit of RNA includes nine highly differentiated regions (V1-V9), which enables the bacterial species to be distinguished $[15,16]$. Classical microbiological methods used to identify bacteria or fungi strains, such as microbiological culture, are ineffective in the case of the human microbiome, as the majority of bacterial strains in the intestine cannot be cultured in laboratory conditions. It has been accepted that the total number of genes of all microorganisms of the human intestinal microbiome exceeds 150 -fold the number of genes in the human genome. The number of bacterial cells existing in the human digestive tract of the healthy individual reaches 100 trillion, 10 times more than the number of cells of the human body $[17,18]$.

Although bacteria account for $99.9 \%$ of the digestive tract microbiome, also two other groups of microorganisms, viruses and fungi, perform important functions. Particular attention should be paid to fungi, which are usually considered as pathogens, although they physiologically colonize skin, the oral cavity as well as other parts of the digestive tract $[19,20]$. The role of fungi in the human microbiome has not been fully recognized, as the first publication on this subject, based on molecular identification methods, appeared in 2006 [21]. Although the Candida genus is most common in the digestive tract [20], the intestinal microbiome, under physiological conditions, can contain more than 50 genera of fungi, most common among them being Candida, Saccharomyces and Cladosporium $[22,23]$. Contrary to the bacterial component of the human intestinal microbiome, the presence of fungi in the microbiome is not permanent and stable [24], and has a rather transient character. However, the results of experimental research suggest that the presence of the fungi plays a large role in the regulation of the human immune system. An example of such influence is the presence of antibodies against Saccharomyces cerevisiae (ASCA) in the majority of sera of Crohn's disease patients [25].

FMT is not limited to the transplantation of the microbiome only. It also includes microparticles, i.e. fragments of chitin ( $\beta-1,4-\mathrm{N}$-acetylglucosamine polymer) - a substance produced naturally by fungi and insects. Chitin microparticles, 1-10 microns in diameter, have been demonstrated to display a strong immunomodulatory influence in experimental models of bowel inflammation [26] and in vitro, affecting human monocytes, which take part in the inflammatory process [27].

Clinical use of FMT can be widespread, although presently the only commonly accepted indication for the use of FMT is Clostridium difficile infection.

\section{Inflammatory bowel disease caused by Clostridium difficile}

In their systematic review, Camaromota et al. [1] analyzed the results from 20 publications, including one randomized study, along with 15 case reports. Almost all patients included in the review were qualified for the study, due to the recurrence of the Clostridium infection after previous, unsuccessful therapy with standard drugs: metronidazole and vancomycin. In the group of 536 patients to whom FMT was applied, 467 were cured (87\%). It was established that the result of the therapy depends on the way of administration of the transplant: to the stomach (81\%), to the duodenum (86\%), to the right part of the colon during the colonoscopy (93\%), or to the left part of the colon via deep enema.

\section{Inflammatory bowel disease}

The group of inflammatory bowel diseases consists primarily of Leśniowski-Crohn's disease and colitis ulcerosa. In patients affected by these conditions, serious defects of the composition and variability of the intestinal microbiome have been observed, mainly in the form of the decreased participation of Firmicutes and Bacte- 
roides species in the microbiome [28]. It is believed that the defects of the microbiome composition lead to the decreased production of short-chain fatty acids [29] butyrates produced by Faecalibacterium prausnitzii in particular [30], which indirectly and directly (through the inhibition of interleukin 8) inhibit inflammatory processes in the intestinal mucosa. The lack of presence of Faecalibacterium prausnitzii was confirmed in Crohn's disease patients.

Currently, clinical data are insufficient for FMT to be recognized as effective in the treatment of inflammatory bowel disease. In a systematic review published in 2015, based on 45 publications [31,32], the effects of the therapy in 112 patients with inflammatory bowel disease (including 6 patients with Crohn's disease) were evaluated. In inflammatory bowel disease, remission was achieved - depending on the institution performing the research - in 0 to $68 \%$ of cases. Such results are not encouraging. Still, it has to be considered that effectiveness of the FMT treatment of inflammatory bowel disease is dependent on the diversity of the donor's microbiome. This may account for the ambivalence of the results of the study.

\section{Irritable bowel syndrome}

Irritable bowel syndrome is a non-inflammatory disease of the intestines, of which abdominal pain and alteration of bowel habits - in the form of both diarrhoea and constipation - are primary symptoms.

Numerous defects in the composition of the intestinal microbiome have been observed in patients affected with this syndrome, as compared to the healthy population [33]. These defects include the presence of Lactobacillus [34] in the faeces of irritable bowel syndrome patients. Some conclude that, due to defects of the intestinal microbiome composition, the production of butyrates decreases, similarly to the situation in the inflammatory intestinal diseases, and the production of hydrogen sulfide increases [35], the latter being capable of stimulating pain receptors in the mouse colon. Clinical data concerning the effectiveness of FMT in the treatment of irritable bowel syndrome are scarce. In the systematic review by Rossen et al. [31] results of the treatment of 13 patients were included. Of that group withdrawal of symptoms was achieved in $70 \%$ of cases. In the research based on a group of 45 patients [36], improvement in the form of normalization of bowel habits was achieved in $60 \%$ of patients, the observation time ranging from 9 to 19 months.

\section{Rheumatoid arthritis}

The modification of the microbiome is gaining in significance in rheumatoid arthritis (RA) as well. It has been established [37] that in RA patients periodontitis associated with the oral microbiome occurs more often. It has been assumed that Porphyromonas gingivalis, a bacterium commonly present in diseases of the periodontium, plays a vital role in early RA $[38,39]$. Preliminary information on the coexistence of intestinal microbiome defects and of early RA was published in 2008 [40]. Numerous experiments have shown the significant influence of modification of the intestinal microbiome on the elements of the inflammatory cascade in arthritis [41]. It has been demonstrated [42] that in the intestinal microbiome of RA patients, there is a significantly higher percentage of Lactobacillus species, compared to the microbiome of healthy individuals.

\section{Type 2 diabetes and obesity}

In patients with type 2 diabetes a number of changes in the composition of the intestinal microbiome, as well as in the metabolic processes that intestinal bacteria take part in, were observed. The increased production, as well as absorption, of short-chain fatty acid produced by gut microbiota has been noted in obese in as compared to slimmer individuals [43]. Changes in the microbiome composition have been observed, consisting in the reduced proportion of Bacteroides species and increased proportion of Firmicutes in it; additionally, in obese persons, an increased proportion of Bifidobacteria has been reported [44]. A particular role has been attributed to specific strains of the Lactobacillus genus. Of this group, in slim individuals, L. gasseri and L. plantarum can be found, while the quantity of Lactobacillus reuteri shows an almost linear correlation with body mass in$\operatorname{dex}[45]$.

\section{Summary}

Recent achievements in the field of genetics allow the exploration of the microbial intestinal environment, which accompanies the human organism from birth. Abundant data suggest that manipulation of the human microbiome content may contribute to the improvement of the treatment results of certain conditions. However, the lack of controlled clinical trials does not allow for the introduction of FMT or its modifications in the treatment of diseases other than Clostridium difficile caused colitis.

The authors declare no conflict of interest.

\section{References}

1. Cammarota G, lanitro G, Gasbarrini A. Fecal microbiota transplantation for the treatment of Clostridium difficile infection. 
A systematic review. J Clin Gastroenterol 2014; 48: Suppl. 1: S80-S84.

2. Rahier JF, Maga F, Abreu C, et al. Second European evidence-based consensus on the prevention, diagnosis and management of opportunistic infections in inflammatory bowel diseases. J Crohns Colitis 2014; 8: 443-468.

3. Yatsusenko T, Rey FE, Manary MJ, et al. Human gut microbiome viewed across age and geography. Nature 2012; 486: 222-227.

4. Claesson MJ, Cusack S, O'Sullivan O, et al. Composition, variability and temporal stability of the intestinal microbiota in the elderly. Proc Natl Acad Sci U S A 2011; 108: 4586-4591.

5. Tyakht AV, Alexeev DG, Popenko AS, et al. Rural and urban microbiota. Gut Microbes 2014; 3: 1-6.

6. Biasucci G, Benenati B, Morelli L, et al. Cesarean delivery may affect the early biodiversity of the intestinal bacteria. J Nutr 2008; 138: 1796S-1800S.

7. Sella DA, Mills DA. Nursing our microbiota: molecular linkages between bifidobacteria and milk oligosaccharides. Trends Microbiol 2010; 18: 298-307.

8. De Filipo C, Cavalieri D, Di Paola M, et al. Impact of diet in shaping gut microbiota revealed by a comparative study in children from Europe and rural Africa. Proc Natl Acad Sci U S A 2010; 107: 14691-14696.

9. Dethlefsen L, Huse S, Sogin ML, et al. The pervasive effects of an antibiotic on the human gut microbiota, as revealed by deep 16S rRNA sequencing. PLoS Biol 2008; 6: e280.

10. Fung TC, Artis D, Sonnenberg GF. Anatomical localization of commensal bacteria in immune cell homeostasis and disease. Immunol Rev 2014; 260: 35-49.

11. Davila AM, Blachier F, Gotteland M, et al. Intestinal luminal nitrogen metabolism: role of the gut microbiota and consequences for the host. Pharmacol Res 2013; 68: 95-107.

12. Vollard EJ, Clasener HAL. Colonisation resistance. Antimicrob Agents Chemiother 1994; 38: 409-414.

13. Lozupone CA, Stombaugh J, Gordon J, et al. Diversity, stability and resilience of the human gut microbiota. Nature 2012; 489: 220-230

14. Moore-Connors JM, Dunn KA, Bielawski JP, et al. Novel strategies for applied metagenomics. Inflamm Bowel Dis 2015; 22: 709-718

15. Wang Y, Qian PY. Conservative fragments in bacterial $16 \mathrm{~S}$ rRNA genes and primer design for $16 \mathrm{~A}$ ribosomal SNA amplicons in metagenomic studies. PLoS One 2009; 4: e7401.

16. Konstantinidis KT, Ramette A, Tiedje JM. The bacterial species definition in the genomic era. Phils Trans R Soc Lond B Biol Sci 2006; 361: 1929-1940.

17. Backhed F, Ley RE, Sonnenburg JL, et al. Host-bacterial mutualism in the human intestine. Science 2005; 307: 1915-1920.

18. Sommer F, Backhed F. The gut microbiota: masters of host development and physiology. Nat Rev Microbiol 2013; 11: 227 238.

19. Richard ML, Lamas B, Liquori G, et al. Gut fungal microbiota: The Yin and Yang of inflammatory bowel disease. Inflamm Bowel Dis 2015; 21: 656-665.

20. Hube B. From commensal to pathogen: stage- and tissue-specific gene expression of Candida albicans. Curr Opin Microbiol 2004; 7: 336-341.
21. Kuhbacher T, Ott SJ, Helwig U, et al. Bacterial and fungal microbiota in relations to probiotic therapy (VLS\#3) in pouchitis. Gut 2006; 55: 1183-1193.

22. Iliev ID, Funari VA, Taylor KD, et al. Interactions between commensal fungi and the C-Type lectin receptor dectin-1 influence colitis. Science 2012; 336: 1314-1317.

23. Hoffmann C, Dollive S, Grunberg S, et al. Archaea and fungi of the human gut microbiome: correlations with diet and bacterial residents. PLoS One 2013; 8: e66019.

24. Dollive S, Chen YY, Grunberg S, et al. Fungi of the murine gut: episodic variations and proliferation during antibiotic treatment. PLoS One 2013; 19: e71806.

25. Quinton JF, Sendid B, Reumaux D, et al. Anti-Saccharomyces cerevisiae mannan antibodies combined with antineutrophil cytoplasmic autoantibodies in inflammatory bowel disease: prevalence and diagnostic role. Gut 1998; 42: 788-791.

26. Nagatani K, Wang S, Llado V, et al. Chitin microparticles for the control of intestinal inflammation. Inflamm Bowel Dis 2012; 18: $1698-1710$

27. Rizzetto L, Ifrim DC, Moretti S, et al. Fungal chitin induces training immunity in human monocytes during cross-talk of the host with Saccharomyces cerevisiae. J Biol Chem 2016 Feb 17. pii: jbc.M115.699645.

28. Damman CJ, Miller SI, Surawicz CM, et al. The microbiome and inflammatory bowel disease: is there a therapeutic role for fecal microbiota transplantation? Am J Gastroenterol 2012; 107: 1452-1459.

29. Machiels K, Joossens M, Sabino J, et al. A decrease of the butyrate-producing species Roseburia hominis and Faecalibacterium prausnitzii defines dysbiosis in patients with ulcerative colitis. Gut 2014; 63: 1275-1283.

30. Tedelind S, Westberg F, Kjerrulf M, et al. Anti-inflammatory properties of the short-chain fatty acids acetate and propionate: a study with relevance to inflammatory bowel disease. World J Gastroenterol 2007; 13: 2826-2832.

31. Rossen NG, McDonald JK, deVries EM, et al. Faecal microbiota transplantation as novel therapy in gastroenterology: A systematic review. World J Gastroenterol 2015; 21: 5359-5371.

32. Vermeire $S$, Joossens $M$, Verbeke K. Donor species richness determines faecal microbiota transplantation success in inflammatory bowel disease. J Crohns Colitis 2015 Oct 29. pii: jjv203 (ahead of publication ).

33. Chassard C, Dapoigny M, Scott KP, et al. Functional dysbiosis within the gut microbiota of patients with constipated-irritable bowel syndrome. Aliment Pharmacol Ther 2012; 35: 828-838.

34. Ponnusamy K, Choi JN, Kim J, et al. Microbial community and metagnomic comparison of irritable bowel syndrome faeces. J Med Microbiol 2011; 60(Pt 6): 817-827.

35. Matsunami M, Tarui T, Mitani K, et al. Luminal hydrogen sulfide plays a pronociceptive role in mouse colon. Gut 2009; 58 : 751-761.

36. Vrieze A, de Groot PF, Kootte RS, et al. Fecal transplant: a safe and sustainable clinical therapy for restoring intestinal microbial balance in human disease? Best Pract Res Clin Gastroenterol 2013; 27: 127-137.

37. dePablo P, Dietrich T, McAlindon TE. Association of periodontal disease and tooth loos with rheumatoid arthritis in the US population. J Reumatol 2008; 35: 70-76. 
38. Scher JU, Ubeda C, Equinda M, et al. Peridontal disease and the oral microbiota in new-onset rheumatoid arthritis. Arthritis Rheum 2012; 64: 3083-3094.

39. deAquino SG, Abdollahi-Roodsaz S, Koenders MI, et al. Peridontal pathogens directly promote autoimmune experimental arthritis by inducing a TLR2- and IL-Driven Th17 response. J Immunol 2014; 192: 4103-4111.

40. Vaahtovuo J, Mnukka E, Korkeamaki M, et al. Fecal microbiota in early rheumatoid arthritis. J Rheumatol 2008; 35: 15001505.

41. Rogier R, Koenders MI, Abdollahi-Roodsaz S. Toll-like receptor mediated modulation of $\mathrm{T}$ cell response by commensal intestinal microbiota as a trigger for autoimmune response for autoimmune arthritis. J Immunol Res 2015; 2015: 527696.

42. Liu X, Zou Q, Zeng Y, et al. Analysis of fecal lactobacillus community structure in patients within early rheumatoid arthritis. Curr Microbiol 2013; 67: 170-176.

43. Schwiertz A, Taras D, Schafer K, et al. Microbiota and SCFA in lean and overweight healthy subjects. Obesity 2010; 18: 190-195.

44. Angelakis E, Armougom F, Million M, Raoult D. The relationship between gut microbiota and weight gain in humans. Future Microbiol 2012; 7: 91-109.

45. Million M, Angelakis E, Maraninchi M, et al. Correlation between body mass index and gut concentrations of Lactobacillus reuteri, Bifidobacterium animalis, Methanobrevibacter smithii and Escherichia coli. Int J Obes 2013; 37: 1460-1466. 